American Journal of Pharmaceutical Education 2017; 81 (5) Article 84.

\title{
RESEARCH
}

\section{Professional Stereotypes of Interprofessional Education Naive Pharmacy and Nursing Students}

\author{
Maria Miller Thurston, PharmD, Melissa M. Chesson, PharmD, Elaine C. Harris, MS, \\ Gina J. Ryan, PharmD \\ Mercer Health Sciences Center, Mercer University, Atlanta, Georgia \\ Submitted January 26, 2016; accepted April 4, 2016; published June 2017.
}

Objective. To assess and compare interprofessional education (IPE) naive pharmacy and nursing student stereotypes prior to completion of an IPE activity.

Methods. Three hundred and twenty-three pharmacy students and 275 nursing students at Mercer University completed the Student Stereotypes Rating Questionnaire. Responses from pharmacy and nursing students were compared, and responses from different level learners within the same profession also were compared.

Results. Three hundred and fifty-six (59.5\%) students completed the survey. Pharmacy students viewed pharmacists more favorably than nursing students viewed pharmacists for all attributes except the ability to work independently. Additionally, nursing students viewed nurses less favorably than pharmacy students viewed nurses for academic ability and practical skills. There was some variability in stereotypes between professional years.

Conclusion. This study confirms the existence of professional stereotypes, although overall student perceptions of their own profession and the other were generally positive.

Keywords: Interprofessional education, professional stereotypes, pharmacy students, nursing students

\section{INTRODUCTION}

As the delivery of health care becomes more interconnected among disciplines, coordinating care between health care professionals is becoming increasingly important. Teaching health profession students how to practice successfully as a member of a multidisciplinary team has quickly become a priority of many educational institutions. Interprofessional education (IPE) is defined by the World Health Organization (WHO) as "when two or more professions learn about, from and with each other to enable effective collaboration and improve health outcomes." According to WHO, by implementing interprofessional collaboration and learning to work together and respect one another's perspectives on health care, multiple disciplines can work more effectively as a team to improve patient outcomes. The Institute of Medicine (IOM) also endorses IPE as a means to improve health care quality. ${ }^{2}$ Furthermore, IPE is required by the Accreditation Council for Pharmacy Education (ACPE), which

Corresponding Author: Melissa M. Chesson, Mercer University College of Pharmacy, Mercer Health Sciences Center, Office No. 131, DuVall Building, 3001 Mercer University Drive, Atlanta, GA 30341-4415. Tel: 678-5476123. Fax:678-547-6384. E-mail: Chesson_mm@mercer. edu increased emphasis on IPE in the Standards $2016 .^{3}$ "Interprofessional communication and collaboration for improving patient health outcomes" is the sixth "essential" element for accreditation of bachelor of science in nursing (BSN) programs. ${ }^{4}$ Both accreditation bodies also support the core competency domains for interprofessional practice, which are detailed by the Interprofessional Education Collaborative (IPEC). They include values/ethics for interprofessional practice, roles/responsibilities, interprofessional communication, and teams and teamwork. ${ }^{5}$ Perceived roles and responsibilities of health care professionals can be influenced by stereotypes or social perceptions that are commonly held about a group, but often oversimplified, prejudice or judgmental. ${ }^{6}$ These professional stereotypes may subsequently impact communication and teamwork.

An early paper examining professional stereotypes in an interprofessional setting included nursing and pharmacy students enrolled in a novel course, "Images of the Health Professions in the Media," and was published in 1987; however, no specific formal evaluation of stereotypes was conducted. ${ }^{7}$ Since that time, various studies have evaluated interprofessional stereotypes of health care professionals $^{8}$ and students ${ }^{9-19}$ using more formalized methods, but only select studies involved both nursing and pharmacy students. ${ }^{9-13,16,17}$ While some studies 


\section{American Journal of Pharmaceutical Education 2017; 81 (5) Article 84.}

assessed only the presence of baseline stereotypes, ${ }^{12,13,16,18}$ others evaluated the impact of IPE interventions of various types and durations in regards to changing baseline professional stereotypes, with mixed results noted..$^{8-11,14,15,17,19}$ Furthermore, while all studies evaluated heterostereotypes or stereotypes of other professions, autostereotypes, also known as stereotypes of one's own profession, were analyzed by merely approximately half of the studies. ${ }^{8,9,11,13,16,18}$

Student stereotypes may be assessed by various methods, including the Health Team Stereotype Scale (HTSS), the Attitudes to Health Professions Questionnaire (AHPQ), the Health Care Stereotypes Scale, and the Student Stereotypes Rating Questionnaire (SSRQ). ${ }^{20}$ In 2014, investigators conducted a systematic review ${ }^{20}$ that evaluated 13 studies involving stereotypes among health care students, two of which utilized the SSRQ and also included nursing and pharmacy students. ${ }^{9,12} \mathrm{Au}-$ thors identified various positive and negative stereotypical adjectives, including ones of nursing and pharmacy students and practitioners. More positive ratings of a student's own profession, compared to another, were noted in studies that included a self-assessment component. ${ }^{20}$ Most recently, investigators have developed a validated Interprofessional Attitudes Scale (IPAS) to assess all four of the IPEC competency domains aforementioned. ${ }^{17}$

It is imperative that students identify and dispel inaccurate perceptions and stereotypes of other professions in order for them to be able to work together on interprofessional teams to provide collaborative patient-centered care after graduation from health science programs. Stereotypes may affect interprofessional communication and impede a team's ability to work together. ${ }^{20}$ Although humans have a natural tendency to categorize information and sometimes use stereotyping to do so, this common process does not yield reliable results. ${ }^{21}$ If health care profession students rely on stereotypes to help direct interprofessional interactions in unfamiliar situations, inaccurate or negative expectations may develop. ${ }^{22}$ Recognition and acknowledgement of professional stereotyping can enhance professional relationships. Identifying commonly held stereotypes among professionals can be useful in IPE curriculum development. Then programs can design IPE activities that address the potentially inaccurate stereotypes commonly held by health professions students that may influence interprofessional interactions before they become engrained. ${ }^{12}$ Furthermore, integration of interprofessional learning experiences into the health professions curricula is essential for student learning and development as well as patient care. $^{23}$ The purpose of this study is to assess baseline nursing and pharmacy student stereotypes of their own profession (autostereotype) and of the other profession (heterostereotype) in a US-based program prior to participation in an interprofessional education activity and to build upon the existing body of knowledge related to health care student professional stereotypes. Furthermore, the stereotypes held by first- vs second-year pharmacy, as well as junior vs senior level nursing students, will be compared.

\section{METHODS}

This study was conducted on Mercer University's Health Sciences Center (MHSC) Atlanta campus, which is comprised of the College of Pharmacy (COP), College of Nursing, and College of Health Professions, which offers physician assistant, physical therapy, clinical medical psychology, and public health programs. The pharmacy program is a four-year doctorate degree program in which experiential education begins in the second semester of the second year, after the point in time of this study. First-year pharmacy students complete approximately 40 hours of simulation-based practical experience, and second-year pharmacy students are provided with 16 additional hours. These hours are completed during the fall semester of each year. At the time of the study, students in each year had completed approximately half of the hours for the semester. The nursing program is a four-year baccalaureate degree program with two years of prerequisites in which the last two years are concentrated solely on the BSN curriculum. Junior level students are provided approximately 100 hours of simulation-based practical experiences, and senior level students receive more than 500 hours of direct patient care clinical practice prior to the time of this study.

In an effort to introduce and reinforce the concept of IPE, COP students are required to attend various largescale IPE activities throughout the curriculum. At the time of the study, the MHSC IPE curriculum included four large-scale events, with one per semester, starting the second year for pharmacy and the junior level for BSN. These events included up to five different health disciplines, with at least three of the following groups participating in each activity: pharmacy, BSN, physician assistant, physical therapy, and nurse practitioner. During fall of the second year, pharmacy students reviewed six case vignettes and identified the relevant provision within their code of ethics (ethics case). Students noted similarities and differences between professions and discussed how to address each dilemma. During spring semester of the same year, students were divided into interdisciplinary teams to complete activities that emphasize the importance of professionalism in practice. Finally, during a pharmacy student's third professional year, interdisciplinary 


\section{American Journal of Pharmaceutical Education 2017; 81 (5) Article 84.}

student teams developed a care plan for a clinical case during fall and spring semesters. Nursing students also participated in all the aforementioned cases, with the exception of the fall clinical case involving third-year pharmacy students.

The participants in this study were health profession students of MHSC $(n=598)$ scheduled to participate in an IPE event. Participants included first-year pharmacy $(\mathrm{n}=174)$, second-year pharmacy $(\mathrm{n}=149), \mathrm{BSN}$ junior $(n=137)$, and BSN senior $(n=138)$ students. At the time of data collection, the aforementioned students had not yet participated in any formal IPE activities, as noted in the previously discussed IPE curriculum. Because these students had not participated in any formal IPE activities as part of the MHSC curriculum, these students were deemed "IPE naive." Students were asked to complete a survey involving the SSRQ to obtain baseline stereotype data for each profession, including their own. The survey was focused on stereotypes of professionals and not stereotypes of professional students. Students were surveyed using pencil and paper during the early part of the academic year, prior to their first formal IPE program (either the first year introductory IPE activity or the ethics case, depending on professional year) in fall semester of 2013. Data was de-identified and aggregated data was analyzed. Additionally, the study was approved by the Mercer University Institutional Review Board.

As part of a larger survey evaluating students' baseline knowledge of IPE and the roles and responsibilities of health care professionals (not reported), students were asked to complete the SSRQ, which was adapted for our use. ${ }^{12}$ Permission to use the SSRQ instrument was obtained prior to study initiation. The IPE Committee at MHSC selected the SSRQ because of its comprehensive design, ease of use, and rigorous validation. There are several other scales that measure stereotypes among health professionals; however, they each had limitations. The report of the Health Care Stereotype Scale did not include validity and reliability statistics, while the investigators of the Health Team Stereotype Scale also did not report validity data, and the Attributes to Health Profession Questionnaire report did not include a reliability scale. Additionally, there are no published reports of use of any of these questionnaires in pharmacy students.

The SSRQ has been tested in a wider variety of health care professional students, including pharmacy, and reliability and validity scales have been published. ${ }^{20}$ The SSRQ is a published and validated survey instrument that consists of a 5-point Likert-type scale from 1 (very low) to 5 (very high). All students rated their own and the other profession (pharmacy and nursing) on nine characteristics including: academic ability, professional competence, interpersonal skills (ie, warmth, sympathy, communication), leadership abilities, ability to work independently, ability to be a team player, ability to make decisions, practical skills, and confidence. ${ }^{8,12}$ The SSRQ instrument also has been utilized successfully in other studies involving the evaluation of professional stereotypes. $9,10,14,16,18$ A higher score on the SSRQ represents a more positive perception of the profession. ${ }^{12}$ Furthermore, scores can be classified as high, medium, or low using mean ratings: 4.0 or above as high, 3.5 to 3.99 as medium, and 3.49 and below as low. ${ }^{12}$ The summary mean score of all traits has previously been used as an overall indication of the perception of a given profession. ${ }^{9}$

Demographic data were extracted from student records and included: age, sex, race, program/year in training, and grade point average (GPA) at the time of the study.

Data analysis was conducted using IBM SPSS Statistics Version 22. Descriptive statistics were used to evaluate baseline demographics and also to summarize SSRQ ratings (means) for each individual profession. Mean ratings were further classified as high (4.0 and above), medium (3.5 to 3.99) and low (3.49 and below). The MannWhitney $\mathrm{U}$ test for independent samples was used to make comparisons between groups. We used $t$-tests for comparison of summary mean scores between professions, and $p$ values of $<.05$ were considered statistically significant.

\section{RESULTS}

A total of 356 students provided informed consent and completed the survey, 207 from the pharmacy program, and 149 from nursing. The overall response rate was $59.5 \%$, with a $64 \%$ and $54.1 \%$ response rate for pharmacy and nursing, respectively.

Table 1 describes the demographics of the pharmacy and nursing student participants. Both groups were similar in age (mid-20s), but there were a higher percentage of females (90.6\%) and Caucasians (67.1\%) in the nursing group. More pharmacy students participated compared to nursing students. There were similar numbers of first- and second-year pharmacy and junior and senior nursing students, with the junior nursing student group having fewer participants. Additionally, the GPA for pharmacy and nursing students at the time of the study was equal at 3.5.

Figure 1 provides details on the comparison of baseline stereotypes of pharmacists. Pharmacy students ranked pharmacists high for all attributes evaluated, while nursing students ranked pharmacists high for all attributes with the exception of interpersonal skills (medium). Additionally, there were significant differences in how pharmacy students viewed pharmacists compared to how nursing students viewed pharmacists for all attributes except the ability to work independently, $p<.05$. For all 


\section{American Journal of Pharmaceutical Education 2017; 81 (5) Article 84.}

Table 1. Participant Demographics $(\mathrm{N}=356)$

\begin{tabular}{lcc}
\hline Characteristic & $\begin{array}{c}\text { Pharmacy } \\
(\mathbf{n}=\mathbf{2 0 7})\end{array}$ & $\begin{array}{c}\text { Nursing } \\
(\mathbf{n}=\mathbf{1 4 9})\end{array}$ \\
\hline Age (years) Mean (SD) & $25.5(3.6)$ & $26(6.1)$ \\
Proportion Females (\%) & 67.1 & 90.6 \\
Race & $\mathrm{N}(\%)$ & $\mathrm{n}(\%)$ \\
$\quad$ Asian & $52(25.1)$ & $13(8.7)$ \\
$\quad$ Black & $45(21.7)$ & $24(16.1)$ \\
White & $93(44.9)$ & $100(67.1)$ \\
$\quad$ Other & $17(8.2)$ & $12(8.1)$ \\
Year in Program & & \\
$\quad$ First & $104(50.2)$ & $61(40.9)$ \\
$\quad$ Second & $103(49.8)$ & $88(59.1)$ \\
Grade Point Average & $3.5(0.36)$ & $3.5(0.32)$ \\
$\quad$ Mean (SD) & & \\
\hline
\end{tabular}

${ }^{a}$ First $=$ first-year pharmacy or junior nursing; second $=$ second-year pharmacy or senior nursing

items, nursing students rated pharmacists lower than pharmacy students rated their own profession. Both groups rated a pharmacist's interpersonal skills the lowest (pharmacy students $=4.38$; nursing students $=3.66$ ). The highest rating given to pharmacists by both groups was for academic ability (pharmacy students $=4.79$; nursing students $=4.66$ ). Pharmacy students gave pharmacists a higher summary mean score of 4.61 , while nursing students gave pharmacists a summary mean score of 4.29 , although both represent high scores.

Figure 2 depicts the comparison of baseline stereotypes of nurses. Nursing students ranked nurses high for all attributes evaluated with the exception of academic and leadership abilities, while pharmacy students ranked nurses high for all attributes with the exception of leadership ability. Additionally, there were significant differences in how nursing students viewed nurses compared to how pharmacy students viewed nurses for academic ability and practical skills, $p<.05$. For all items, pharmacy students rated nurses higher than nursing students rated their own profession, with the exception of ability to work independently. Both groups rated nurses the lowest in leadership ability (nursing students $=3.79$; pharmacy students $=3.96$ ), while both groups also rated nurses the highest in interpersonal skills (nursing students $=4.54$; pharmacy students $=4.56$ ). Nursing students assigned their own profession a summary mean score of 4.19 , while pharmacy students gave nurses a higher summary mean score of 4.28, both representing high scores.

Figure 3 illustrates the comparison of stereotypes between professional years. There was a difference in how first- vs second-year pharmacy students viewed pharmacists, with first-year students providing lower ratings for all characteristics, $p<.05$, except leadership ability, ability to be a team player, and decision-making ability. However, there was no difference in how junior vs senior nursing students viewed pharmacists for all attributes, $p>.05$. Furthermore, junior nursing students had a higher perception of nurses than senior nursing students, and first-year pharmacy students had a lower perception of nurses than second-year pharmacy students for all attributes, $p<.05$ for all comparisons.

\section{DISCUSSION}

This study confirms the existence of professional stereotypes in pharmacy and nursing students in

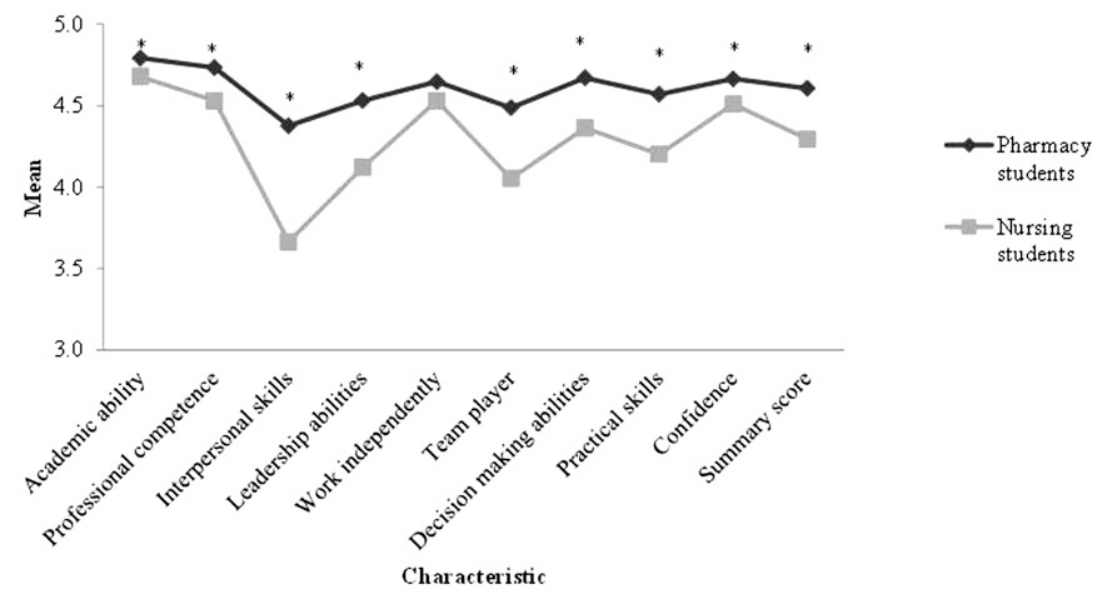

Likert scale key: $1=$ very low and $5=$ very high

Mann-Whitney U performed to derive $p$-values; however, depicted as mean scores to simplify the visual interpretation of data, $* p<.05$.

Figure 1. Comparison of Baseline Stereotypes of Pharmacists. 


\section{American Journal of Pharmaceutical Education 2017; 81 (5) Article 84.}

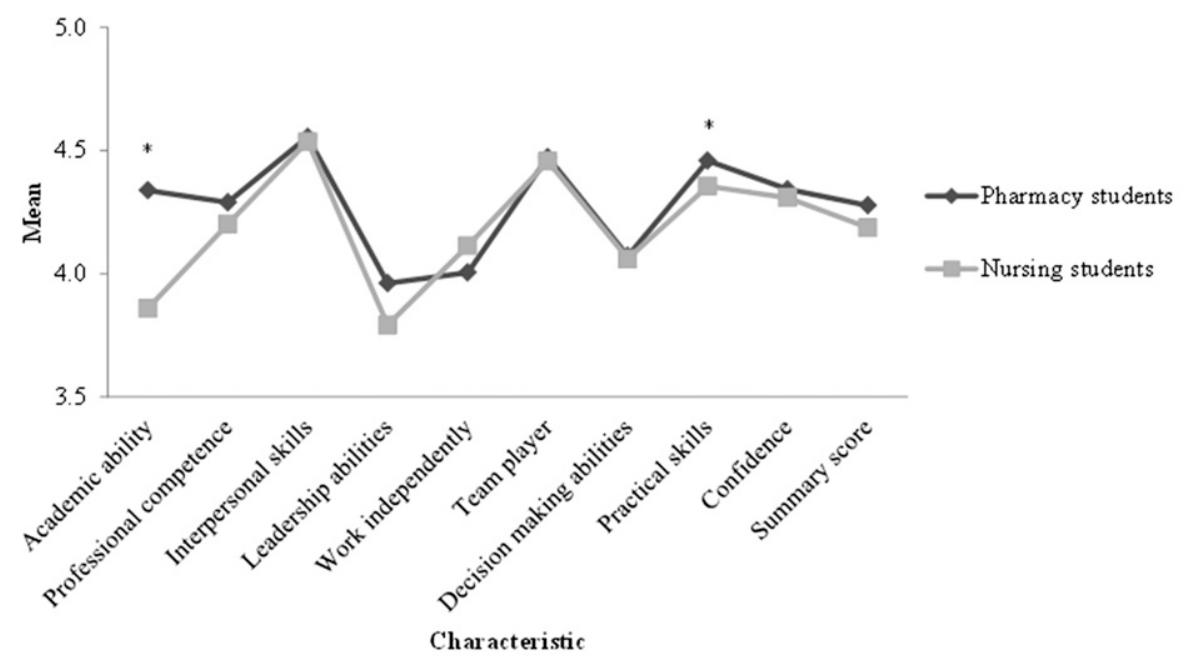

Likert scale key: $1=$ very low and $5=$ very high.

Mann-Whitney U performed to derive $p$-values; however, depicted as mean scores to simplify the visual interpretation of data, $* p<.05$.

Figure 2. Comparison of Baseline Stereotypes of Nurses.

a US-based program, which is consistent with the results of Hean and colleagues, who surveyed students in England. ${ }^{12}$ The presence of professional profiling that was identified demonstrates the cross-cultural relevance and reality of professional stereotypes. Additionally, it adds to the knowledge of autostereotypes as identified by Michalec and colleagues, ${ }^{16}$ but also reports the difference in student stereotypes between professional years. As previously noted, these stereotypes can impact communication in the work environment, which has been shown to affect patient care. ${ }^{20}$ This concept has practical application for our students, as they may be at risk for difficulties with interprofessional collaboration within the clinical setting. The existence of baseline professional stereotypes also was identified several years ago, in a study by Barnes and colleagues, which evaluated community mental health practitioners. ${ }^{8}$ The topics were further investigated later in a population of undergraduate physical therapy and podiatry students. ${ }^{15}$ In 2015 , yet another study evaluating the impact of IPE on moderation of professional stereotypes revealed noticeable differences in the way various professions were perceived at baseline. ${ }^{10}$ There is also evidence supporting the concept that upon matriculation into a professional program, students already have preconceived ideas about other professions. ${ }^{19}$ The pharmacy students in this study were early in their professional careers, as were the junior level nursing students. Additionally, in 2003, it was noted that health care students rated other professions more favorably if they had a strong, positive association with their own profession. ${ }^{13}$ This was also evident in the present study in which pharmacy students generally rated both their own profession and nurses higher than nursing students did. Two additional studies noted that most student groups tended to rate their own profession higher for virtually every quality when compared to others. ${ }^{11,16}$ This "in-group favoritism" may be supported by the social identity theory, in which a student view their future professional group in the most positive manner, potentially at expense to another profession. ${ }^{24}$

While ratings for individual attributes were generally similar between the score given to one's own profession and that of the other profession, it was noted that regarding stereotypes of pharmacists, there were significant differences in all items except ability to work independently. Although a pharmacist's role in the health profession has been expanding and gaining appreciation, limitations on provider status and prescriptive authority indeed hinder a pharmacist from serving as an independent practitioner in most practice settings and this was likely recognized by both student groups. As mentioned above, pharmacy students rated pharmacists higher than nursing students rated pharmacists for all attributes, with the highest rating being academic ability. This high ranking for academic ability was also noted in prior studies evaluating the stereotypes of pharmacists. ${ }^{12,16}$ While there is literature indicating the profession of a physician is associated with academic rigor, our study adds to the body of evidence to support pharmacy also being viewed as a profession of greater intellectual ability. It is unknown how this would influence the interprofessional team; however, it may 


\section{American Journal of Pharmaceutical Education 2017; 81 (5) Article 84.}
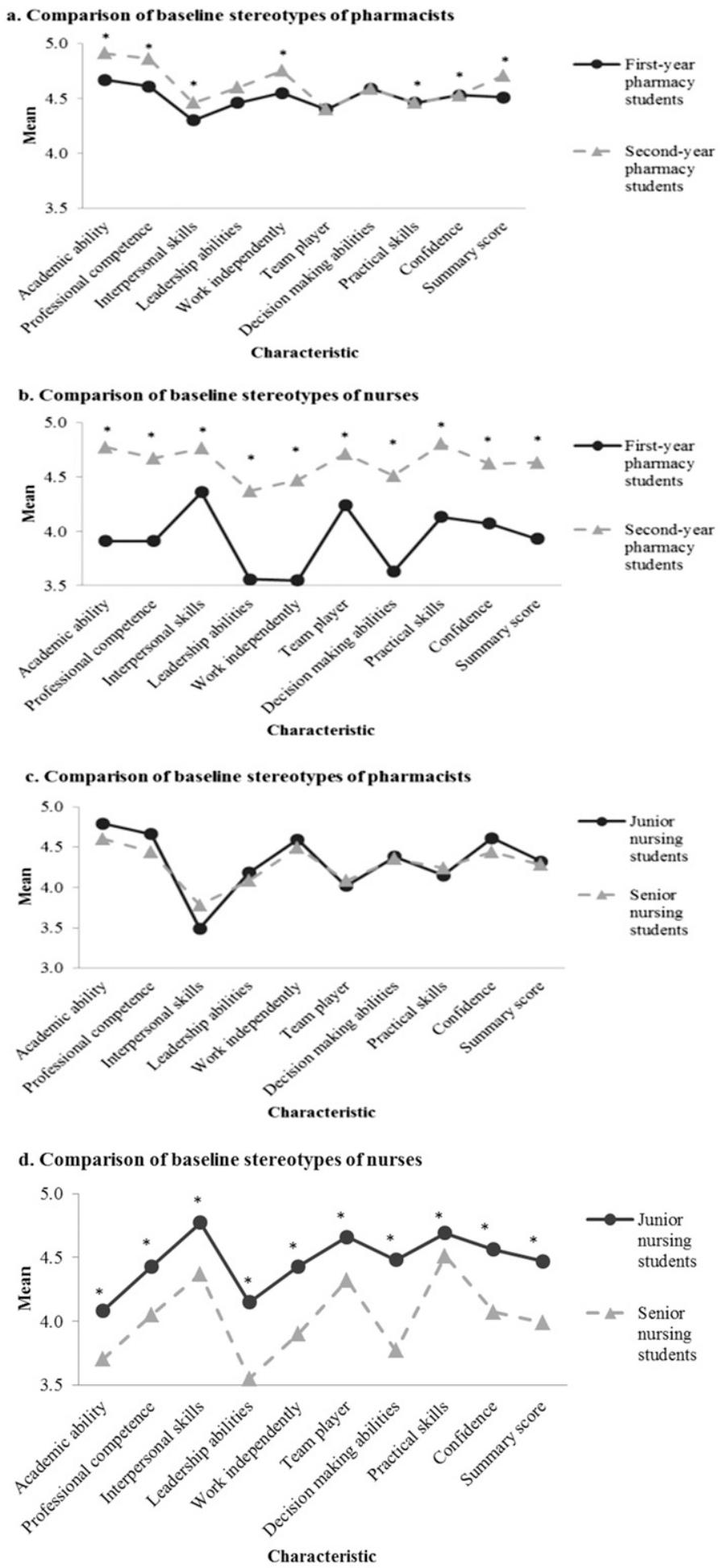

Likert scale key: $1=$ very low and $5=$ very high.

Mann-Whitney $\mathrm{U}$ performed to derive $p$-values; however, depicted as mean scores to simplify the visual interpretation of data, ${ }^{*} p<.05$.

Figure 3. Comparisons of Baseline Stereotypes Between Professional Years.

affect a nurse's ability to assume a leadership role. ${ }^{12}$ These high rankings may be due to the fact that pharmacy students complete a graduate level doctorate degree program and nursing students are undergraduates; however, the GPA at the time of the study for both nursing and pharmacy students was equivalent, indicating that both groups were succeeding academically in their respective programs. 


\section{American Journal of Pharmaceutical Education 2017; 81 (5) Article 84.}

Overall, the attribute ratings for pharmacists were generally higher than for nurses, indicated by a higher summary mean score. The lowest rating for pharmacists overall was interpersonal skills, which is consistent with results from previous studies. ${ }^{8,9,12,13,16,19}$ While first-year pharmacy students in this study had previously completed a communications course at the time of data collection, their leadership course had not yet been taken, which provides some additional content related to communication skills such as emotional intelligence that may further influence their interpersonal skills. Additionally, these students had completed minimal experiential education in a traditional clinical setting. Furthermore, pharmacists have historically had limited interaction with the health care team, and in most interactions the pharmacist is functioning in an auditor-type role (eg, identifying inappropriate drug selection, dosing, or administration). Despite substantial developments in expanding the role of a pharmacist as an integral member of the team who can provide direct patient care, stereotypical views on interpersonal skills appear to prevail. Similarly to the present study, Michalec and colleagues noted that ratings for the attributes of interprofessional skills and ability to be a team player were low for pharmacy, while academic ability was rated highest. ${ }^{16}$ Overall, self-ratings of the noted attributes indicate that pharmacy students may have an overinflated perception of themselves.

In regards to stereotypes of nurses, there were significant differences apparent in academic ability and practical skills with pharmacy students rating nurses higher than nursing students rated themselves. Nurses were rated lower overall by both groups as indicated by the lower summary mean score. This inferior self-rating is not consistent with other studies where nurses rated themselves higher. ${ }^{16}$ However, Sollami and colleagues also detected similar troublesome findings relating to autonomy and competence in that nursing students tended to perceive nurses in a more traditional manner and in a less favorable light. ${ }^{18}$ In our study, the results may be due to the fact that the nursing program is the only undergraduate program in the MHSC. It is possible that undergraduate nursing students may be self-conscious, since pharmacy students are working toward a doctorate degree. This could affect perceptions of self (ie, rating their own profession lower) and a diminished appreciation of their professional role. It has been reported that negative public nursing stereotypes may affect their self-esteem, thus potentially contributing to this finding. ${ }^{25}$ This lesser perception of self also appears to worsen as a nursing student transitions from junior to senior year. However, this "undergraduate" status does not appear to affect what other professions think of them. In fact, second-year pharmacy students exhibited more favorable stereotypes of nurses when compared to first-year students, indicating a gained appreciation of nurses during their professional career, likely as they began to fully understand the vast roles and responsibilities of a nurse.

In regards to specific stereotypes of nurses and as previously mentioned, pharmacy students rated nurses higher than nursing students rated themselves for all attributes with the exception of the ability to work independently. The highest rating was interpersonal skills and lowest leadership abilities. The nursing profession is widely known for its caring nature and has placed this tenet at the core of the practice to better differentiate itself. ${ }^{26}$ The nursing leadership course takes place during the last semester of the curriculum; therefore, nursing students in this study may not yet have developed a full understanding and appreciation of their leadership role at the time of data collection. Although there is not a standalone communications course for nurses, themes of communication and collaboration are consistently integrated throughout the curriculum. Finally, the junior nursing students only had one month of clinical experience. However, senior level nursing students had completed a significant number of hours in acute care settings as discussed in the methods section.

The data indicated that first-year pharmacy students tend to have a lower perception of pharmacists and nurses, compared to second-year pharmacy students. This may be related in part to them having not yet completed their clinical disease state modules. Therefore, the first-year students may not yet realize the complexity of their profession or the nursing profession as well as the full array of attributes necessary to be a successful practitioner. Nursing students, on the other hand, appear to demonstrate a decrease in the perception of nurses from their junior to senior year. As a result of the concentrated nature of the two-year nursing program, nursing students were deeper into the program at the time of this study and may have been able to more quickly realize the vast array of skills necessary to be professional. This experience may have helped them realize knowledge and skill deficits. There is a lack of evidence that evaluates the changes in professional stereotypes that occur as a health profession student advances through the curriculum.

The MHSC IPE Committee used this data to guide the development of an introductory IPE activity that focuses on roles and responsibilities. Additionally, when IPE activities are developed the committee intentionally creates activities where nurses are positioned to lead the group and where pharmacists are required to demonstrate interpersonal skills. 


\section{American Journal of Pharmaceutical Education 2017; 81 (5) Article 84.}

\section{Limitations}

The response rates for pharmacy and nursing students were $64 \%$ and $54 \%$, respectively, and the overall response rate was less than $70 \%$. Furthermore, because this study only included students from one health profession education institution, the results may not be generalizable. This study explores the stereotype relationship between two professional groups, pharmacy and nursing students, while other past studies have evaluated three or more groups. ${ }^{8-13,16}$ However, nursing and pharmacy are professions that tend to work closely together in practice, especially in inpatient settings. Although all students in this study were considered IPE naive, it is not possible to determine all of their past interprofessional interactions, such as work and life experiences outside of the curriculum that could have affected their baseline stereotypes; therefore, it was not possible to control for personal life experiences. Finally, this study does not assess the impact of an IPE intervention on changes in stereotypes, but does provide valuable information about baseline stereotypes that should be addressed in IPE curricula. Other institutions may benefit from assessing stereotypes existing in their student body.

\section{CONCLUSION}

The existence of professional stereotypes in pharmacy and nursing students was identified in this study, within a population of US-trained students at one health sciences program. The SSRQ has been used in multiple health disciplines and with other types of IPE activities as an instrument to evaluate professional stereotypes among health profession students. Identification and recognition of results of such surveys is imperative to target IPE programs to address noted stereotypes/low rated attributes, even though they may be potentially overinflated. Further studies should seek to determine causes of and reasons for existing stereotypes and determine if and how theses stereotypes may affect patient care. It may be beneficial to share such information with students to increase awareness and stimulate and enrich dialogue about the stereotypes commonly held. Student understanding of each other likely will lead to increased collaboration in their future work environment and improvements in patient care and outcomes. Students in this study will continue to be followed to determine if IPE interventions are successful at affecting the previously discussed stereotypes.

\section{REFERENCES}

1. World Health Organization (WHO). Framework for action on interprofessional education \& collaborative practice. Geneva: World Health Organization; 2010. http://apps.who.int/iris/bitstream/10665/ 70185/1/WHO_HRH_HPN_10.3_eng.pdf. Accessed May 28, 2015.
2. Institute of Medicine Committee on the Health Professions Education Summit. Health Professions Education: A Bridge to Quality. Washington, DC: National Academies Press; 2003. http:// www.ncbi.nlm.nih.gov/books/NBK221528/. Accessed October 30, 2015.

3. Accreditation Council for Pharmacy Education. Accreditation standards and key elements for the professional program in pharmacy leading to the doctor of pharmacy degree. Standards 2016. https:// www.acpe-accredit.org/pdf/Standards2016FINAL.pdf. Accessed June 3, 2015.

4. American Association of Colleges of Nursing. The Essentials of Baccalaureate Education for Professional Nursing Practice. Washington, DC: American Association of Colleges of Nursing; 2008. www.aacn.nche.edu/education-resources/BaccEssentials08. pdf. Accessed March 18, 2016.

5. Interprofessional Education Collaborative. Core Competencies for Interprofessional Collaborative Practice: Report of an Expert Panel. Washington, DC: Interprofessional Education Collaborative; 2011. http://www.aacn.nche.edu/education-resources/ipecreport.pdf. Accessed June 1, 2015.

6. Turner JC. Some current issues in research on social identity and self-categorization theories. In: Ellemers N, Spears R, Doosje B, eds. Social Identity, Context, Commitment, Content. Hoboken, NJ: WileyBlackwell; 1999:6-34.

7. Poirier S, Lipetz M. Pharmacy in interprofessional education: a course on images of the health professions in the media. Am J Pharm Educ. 1987;51(2):133-137.

8. Barnes D, Carpenter J, Dickinson C. Interprofessional education for community mental health: attitudes to community care and professional stereotypes. Social Work Education. 2000;19(6):565583.

9. Ateah CA, Snow W, Wener P, et al. Stereotyping as a barrier to collaboration: does interprofessional education make a difference? Nurse Educ Today. 2011;31(2):208-213.

10. Foster R, Macleod Clark J. Moderating the stereotypical views of health and social care students: the role of interprofessional education. J Interprof Care. 2015;29(1):34-40.

11. Hawkes G, Nunney I, Lindqvist S. Caring for attitudes as a means of caring for patients - improving medical, pharmacy and nursing students' attitudes to each other's professions by engaging them in interprofessional learning. Med Teach. 2013;35(7):e1302e1308.

12. Hean S, Clark JM, Adams K, Humphris D. Will opposites attract? Similarities and differences in students' perceptions of the stereotype profiles of other health and social care professional groups. $J$ Interprof Care. 2006;20(2):162-181.

13. Hind M, Norman I, Cooper S, et al. Interprofessional perceptions of health care students. J Interprof Care. 2003;17(1):21-34.

14. Liaw SY, Siau C, Zhou WT, Lau TC. Interprofessional simulation-based education program: a promising approach for changing stereotypes and improving attitudes toward nurse-physician collaboration. Appl Nurs Res. 2014;27(4):258-260.

15. Mandy A, Milton C, Mandy P. Professional stereotyping and interprofessional education. Learn Health Soc Care. 2004;3(3):154170.

16. Michalec B, Giordano C, Arenson C, Antony R, Rose M. Dissecting first-year students' perceptions of health profession groups: potential barriers to interprofessional education. J Allied Health. 2013;42(4):202-213.

17. Norris J, Carpenter JG, Eaton J, et al. The development and validation of the Interprofessional Attitudes Scale: assessing the 


\section{American Journal of Pharmaceutical Education 2017; 81 (5) Article 84.}

interprofessional attitudes of students in the health professions. Acad Med. 2015;90(10):1394-1400.

18. Sollami A, Caricati L, Mancini T. Ambivalent stereotypes of nurses and physicians: impact on students' attitude toward interprofessional education. Acta Biomed. 2015;86 Suppl 1:19-28. 19. Tunstall-Pedoe S, Rink E, Hilton S. Student attitudes to undergraduate interprofessional education. J Interprof Care. 2003;17 (2):161-172.

20. Cook K, Stoecker J. Health care student stereotypes: a systematic review with implications for interprofessional collaboration. $J$ Res Interprof Pract Educ. 2014;4(2):1-13.

21. Haslam SA, Turner JC, Oakes PJ, Reynolds KJ, Doosje B. From personal pictures in the head to collective tools in the world: How shared stereotypes allow groups to represent and change social reality. In McGarty C, Yzerbyt VY, Spears R, eds. Stereotypes as Explanation:
The Formation of Meaningful Beliefs About Social Groups. Cambridge, UK: Cambridge University Press; 2002:157-185.

22. Kirkham M, Stapleton H, Curtis P, Thomas G. Stereotyping as a professional defence mechanism. Br J Midwifery. 2002;10(9):549552.

23. Frenk J, Chen L, Bhutta ZA, et al. Health professionals for a new century: transforming education to strengthen health systems in an interdependent world. Lancet Lond Engl. 2010;376(9756):19231958.

24. Tajfel H, Billig MG, Bundy RP, Flament C. Social categorization and intergroup behaviour. Eur J Soc Psychol. 1971;1(2):149-178. 25. Takase M, Kershaw E, Burt L. Nurse-environment misfit and nursing practice. $J$ Adv Nur. 2001;35(6):819-826.

26. Paley J. Caring as a slave morality: Nietzchean themes in nursing ethics. J Adv Nur. 2002;40(1):25-35. 